\title{
Current Issues on Bioavailability and Bioequivalence Determination
}

\section{Chyung S. Cook*}

Baxter Healthcare, USA

\begin{abstract}
Methodologies for determining bioavailability and bioequivalence of endogenous compounds and macromolecules such as polymers are different from that of small exogenous compounds. In many cases, bioavailability and bioequivalence guidelines for exogenous molecules may not be appropriate for endogenous compounds. A common approach of baseline subtraction for endogenous materials is arguable in some cases, since levels of endogenous materials are highly variable during an experimental period. For polymers molecular weight distribution may be different among the materials from different manufactures and/or different manufacturing processes. These issues are addressed along with following issues regarding bioavailability/ bioequivalence determination: use of pharmacokinetic vs. pharmacodynamic parameters, use of a chiral assay vs. total assay for racemic drugs, and total vs. free concentration assays for drugs that exhibit concentration-dependent plasma protein binding, and for liposome/protein encapsulated formulations.
\end{abstract}

Keywords: Bioavailability; Bioequivalence; Endogenous compounds; Pharmacokinetic-pharmacodynamic relationship

\section{Introduction}

Bioavailability (BA) and bioequivalence (BE) guidelines are relatively well established for evaluation of exogenous compounds following oral administration.However, guidelines specific to the determination of BA and $\mathrm{BE}$ for endogenous compounds are not in place. $\mathrm{BA}$ and $\mathrm{BE}$ determination of endogenous compounds is substantially different from that of exogenous compounds. For endogenous compounds there exist baseline levels of the target analyte without administering the test compounds, and the baseline of the endogenous substances are not constant during an experimental period. Therefore, in many cases BA and BE guidelines of exogenous molecules may not be appropriate for endogenous compounds, especially with regard for proteins/peptides. Therefore, issues often surround the justification of an appropriate method for baseline subtraction with endogenous compounds. In this presentation issues associated baseline subtraction are addressed along with the discussion of current issues regarding $\mathrm{BA}$ and $\mathrm{BE}$ as follows: $\mathrm{BE}$ determination of intravenous (IV) formulations, use of pharmacokinetic vs. pharmacodynamic parameters for BA and $\mathrm{BE}$ estimation, application of chiral assays, and reliance on total vs. free concentrations.

\section{IV formulations}

Currently, FDA guidelines for IV formulations say "A drug product's in vivo $\mathrm{BA}$ or $\mathrm{BE}$ may be considered self evident if the product is a parental solution intended solely for administration by injection, or an ophthalmic or otic solution; and contains the same active and inactive ingredients in the same concentrations as a drug product that is the subject of an approved full new drug application or abbreviated new drug application [1]." A question may arise why a BA/BE study for an IV formulation is necessary since BA of a test formulation will always be $100 \%$, regardless of formulation. However, substantially different IV pharmacokinetic characteristics are expected even after IV administration in the following situations:new excipients in a test formulation which may affect excretion, metabolism and/ or distribution (e.g., acidic and basic compounds, metabolism inhibitors), nanosuspensions, liposomal or protein encapsulated formulations with different dissolution rates,different salts with different molecular weights and polymers with different molecular weight distributions. Therefore, depending upon the situation, a BA/BE study may be required after IV administration. Presented in Table 1 are molecular weights and elimination half-lives in humans following IV administration of various carbohydrate salts of iron [2] Molecular weights of iron salts are different and range from $38 \mathrm{KD}$ to $238 \mathrm{KD}$. Even for the same iron dextran salt, molecular weight is substantially different depending upon the manufacturer and the resulting pharmacokinetics of these dextran salts are different as shown in Table 1. Another example is that iron sucrose obtained from two different manufacturers was not bioequivalent following IV infusion (Figure 1) [3]. This appeared to be due to differences in molecular weights and other physicochemical characteristics. Therefore, when $\mathrm{BA} / \mathrm{BE}$ of polymers and macromolecules are to be evaluated, careful consideration for the determination of physicochemical properties prior to pharmacokinetic studies is important.

\section{Endogenous compounds}

Plasma concentrations of endogenous compounds are generally not constant and have been shown to be highly variable within and between days. When serum levels of endogenous iron concentrations

\begin{tabular}{|c|c|c|}
\hline Compound & MW (KD) & $\mathrm{t}_{1 / 2}(\mathrm{hrs})$ \\
\hline Iron dextran (Dexferrum) & 265 & $\sim 60$ \\
\hline Iron dextran (Imferon) & 73,103 & $\sim 50$ \\
\hline Iron dextran (INFeD) & 96 & $\sim 50$ \\
\hline Iron Sucrose & 43 & 6 \\
\hline Ferric Gluconate & 38 & 1.3 \\
\hline
\end{tabular}

Table 1: Elimination half-life of various iron salts.

*Corresponding author: Chyung S. Cook, Ph.D., 25212 Illinois Route 120, Round Lake, IL 60073, USA, Tel: (847) 270-5319; E-mail: Chyung_cook@baxter.com

Received August 30, 2011; Accepted September 22, 2011; Published September 24, 2011

Citation: Cook CS (2011) Current Issues on Bioavailability and Bioequivalence Determination. J Bioequiv Availab S1. doi:10.4172/jbb.S1-003

Copyright: ( 2011 Cook CS. This is an open-access article distributed under the terms of the Creative Commons Attribution License, which permits unrestricted use, distribution, and reproduction in any medium, provided the original author and source are credited. 
were determined at frequent intervals (every 2 hours) in the dog respective iron levels were reduced drastically from $90 \mathrm{mg} / \mathrm{dL}$ to $10 \mathrm{mg} / \mathrm{dL}$ during an approximate 10-hour period (Figure 2). In this study, the reason for this decrease in blood levels of iron appeared to be due in part to the depletion of endogenous iron by frequent blood sampling. However, subtraction of predose levels in this case may have been extremely biased and even resulted in negative AUCs, regardless of any suspected depletion mechanism involved. Further investigations in humans showed that iron concentrations were not constant over a $24 \mathrm{hr}$ period while concentrations of total iron binding capacity, transferrin saturation and ferritin were relatively constant over the same period (Table 2) [4]. No consistent pattern was observed in within-day variations of iron concentrations.Variations of iron

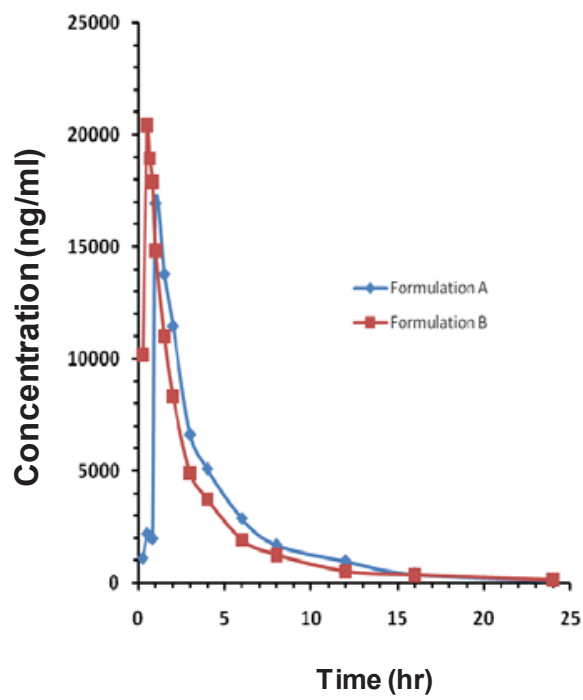

Figure 1: Mean serum concentration of iron sucrose in man (Cook et al., unpublished data). concentrations were shown to be highly dependent on the individual, and no consistent pattern in inter-day variations was observed (Figure 3) [4]. These results indicate that subtraction of predose levels for BA/ $\mathrm{BE}$ determination of endogenous compounds may not be appropriate in some cases.Then the question is "can we use total (endogenous plus exogenous) concentrations instead of baseline subtracted exogenous concentrations for BA/BE determination?" AUC ratios of total material for the test to the reference formulations, $\left(\mathrm{AUC}_{\mathrm{tot}}\right)_{\text {test }}$ I $\left(\mathrm{AUC}_{\text {tot }}\right)_{\text {ref }}$ will change depending upon the extent of $\mathrm{AUC}_{\text {endo }}$ (AUC of endogenous material) contribution to $\mathrm{AUC}_{\text {tot }}$ and may be substantially different from the ratio of baseline-subtracted $\left(\mathrm{AUC}_{\mathrm{exo}}\right)_{\text {test }} /\left(\mathrm{AUC}_{\mathrm{exo}}\right)_{\text {ref }}$ (See Table 3). Ratios of $\left(\mathrm{AUC}_{\mathrm{tot}}\right)_{\text {test }} /\left(\mathrm{AUC}_{\mathrm{tot}}\right)_{\text {ref }}$ will not represent true systemic exposure ratios for comparison of different formulations (Figure 4). When the ratio of $\left(\mathrm{AUC}_{\text {exo }}\right)_{\text {test }} /\left(\mathrm{AUC}_{\text {exo }}\right)_{\text {ref }}$ is less than 1.0the ratio determined using total AUC is greater. When the ratio of $\left(\mathrm{AUC}_{\text {exo }}\right)_{\text {test }} /\left(\mathrm{AUC}_{\text {exo }}\right)_{\text {ref }}$ is greater than 1.0 the ratio determined using total AUC is smaller (Table 3). An example is shown in Figure 5 with corresponding statistical data given in Table 4 [3]. As the contribution of the endogenous material to the total AUC increases, there is the better chance to meet the BE requirement. Therefore the following points may warrant careful consideration for baseline adjustment in $\mathrm{BE}$ assessment: Perform pre- and postdose characterization of endogenous concentrations (preferably at three time intervals) within range of the same blood sampling period. For example, if blood samples were taken for a 12 hour period then the predose blood samples may be taken at 8:00 AM, 12:00 PM and 8:00 PM one day before dosing, as well as blood sampling prior to dosing on the day of dosing. Post study blood samples at the same time points may be taken after blood sampling is completed for the study and/or at the end of each treatment (during a washout period). Determine baseline values for each individual at each dosing interval, if appropriate.Examine if there are any trends in baseline values. In the instance that a trend is observed (decreasing or increasing), constant baseline subtraction may be arguable. For a cross-over study statistical analysis of baseline values at each treatment period may be needed. If possible use of a dose which will minimize contribution of baseline AUC to overall AUC is suggested.

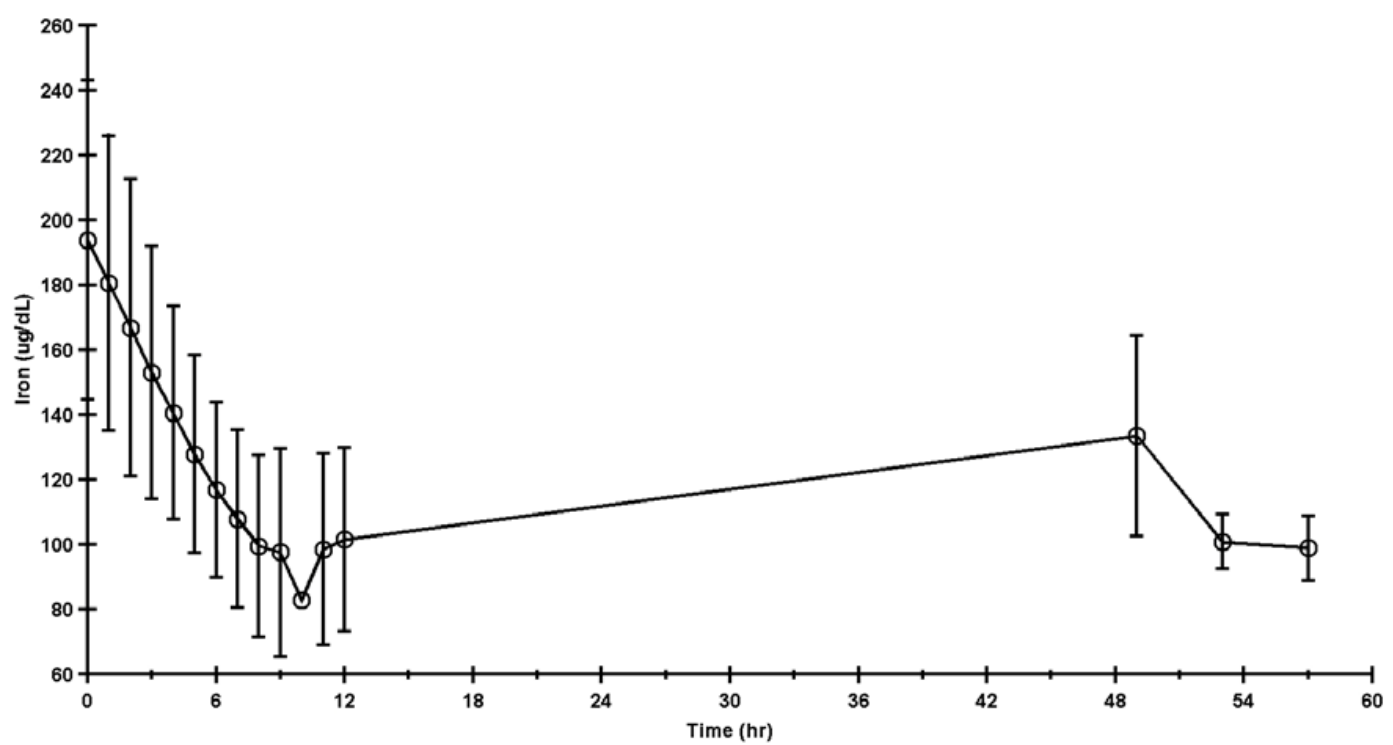

Figure 2: Endogenous serum concentrations of iron in the dog (unpublished data). 


\section{Pharmacokinetic vs. pharmacodynamic parameters}

$\mathrm{BA}$ and BE may be determined using both plasma concentrations of a drug (pharmacokinetic parameters) and its pharmacological effects (pharmacodynamic parameters). However, drug concentrations (C) in blood/plasma, the active site or the peripheral compartment and the pharmacological effects (E) often follow a non-linear relationship, known as the Hill equation (equation below and Figure 6). As a result BA values obtained using pharmacokinetic and pharmacodynamic parameters may be substantially different.

$$
\mathrm{E}=\frac{\mathrm{E}_{\max ^{*}} \mathrm{C}^{\mathrm{n}}}{\mathrm{EC}_{50}^{\mathrm{n}}+\mathrm{C}^{\mathrm{n}}}
$$

$\mathrm{E}_{\max }$ is the maximal effect attributable to the drug, $\mathrm{EC}_{50}$ is the concentration producing $50 \%$ of the maximum effect and $\mathrm{n}$ is Hill's coefficient.

The concentration-effect curve (Figure 6) produced from the application of Hill's equation shows approximate linearity from roughly $20-80 \%$ of the maximum attainable intensity of a response, which is a region of particular interest in drug therapy. At high concentrations of a substrate the pharmacological response may be saturated. Therefore it is important to select a dose in the range of the linear responses when pharmacodynamic parameters are used for BA and BE determination. For more details on this subject, the following chapter in the present issue can be referred: Differences in Relative Bioavailability (BA) of

\begin{tabular}{|c|c|c|c|c|}
\hline \multirow{2}{*}{ Time } & \multicolumn{4}{|c|}{ Analyte } \\
\cline { 2 - 5 } & $\begin{array}{c}\text { Iron } \\
(\mu \mathrm{g} / \mathrm{dL})\end{array}$ & $\begin{array}{c}\text { TIBC } \\
(\mu \mathrm{g} / \mathrm{dL})\end{array}$ & $\begin{array}{c}\text { TS } \\
(\%)\end{array}$ & $\begin{array}{c}\text { Ferritin } \\
(\mu \mathrm{g} / \mathrm{L})\end{array}$ \\
\hline Day1, 8 AM & $90 \pm 37$ & $335 \pm 43$ & $27.3 \pm 11.8$ & $38.1 \pm 38$ \\
\hline Day1, Noon & $98 \pm 36$ & $348 \pm 42$ & $28.4 \pm 0.9$ & $38.8 \pm 38.2$ \\
\hline Day1, 4 PM & $93 \pm 43$ & $346 \pm 41$ & $27.1 \pm 12.3$ & $36.8 \pm 35.8$ \\
\hline Day 2, 8 AM & $95 \pm 32$ & $336 \pm 45$ & $28.7 \pm 10.7$ & $38.4 \pm 37.7$ \\
\hline
\end{tabular}

Table 2: Intra-day variations of mean $( \pm S D)$ concentrations of iron, total iron binding capacity (TIBC), transferrin saturation (TS) and ferritin in man.
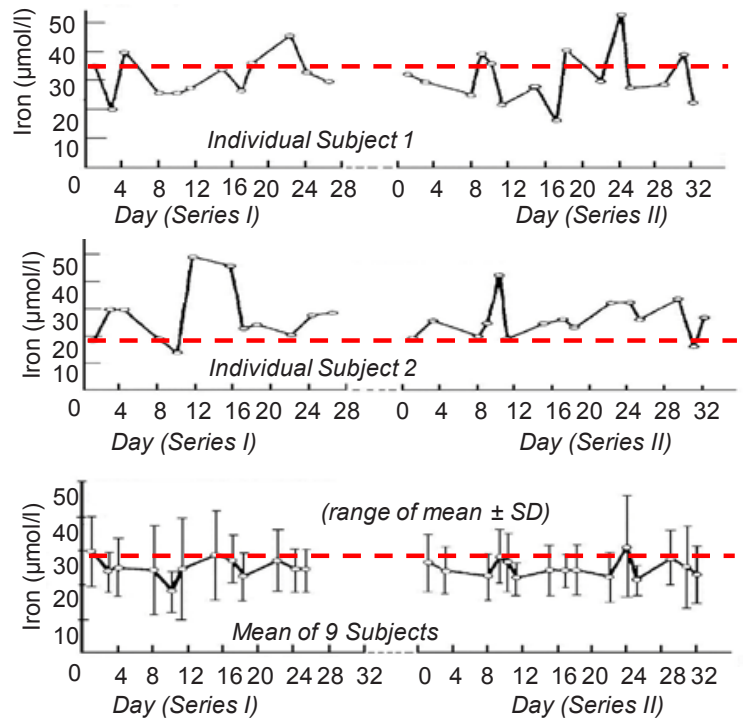

Figure 3: Inter-day variations of iron concentrations.

\begin{tabular}{|c|c|c|c|c|c|}
\hline \multirow{2}{*}{$\left(\mathrm{AUC}_{\text {exo }}\right)_{\text {test }} /\left(\mathrm{AUC}_{\text {exo }}\right)_{\text {ref }}$} & \multicolumn{5}{|c|}{$\mathrm{AUC}_{\text {endo }} / \mathrm{AUC}_{\text {tot }}$} \\
\cline { 2 - 6 } & 0.05 & 0.1 & 0.2 & 0.3 & 0.5 \\
\hline 0.75 & 0.76 & 0.78 & $0.8^{*}$ & $0.83^{*}$ & $0.88^{*}$ \\
\hline 0.8 & 0.81 & 0.82 & 0.84 & 0.86 & 0.9 \\
\hline 1.25 & 1.24 & 1.23 & 1.2 & 1.18 & 1.13 \\
\hline 1.3 & 1.29 & 1.27 & $1.24^{*}$ & $1.21^{*}$ & $1.15^{*}$ \\
\hline 1.5 & 1.48 & 1.45 & 1.4 & 1.35 & $1.25^{*}$ \\
\hline
\end{tabular}

$\left(A \cup C_{\text {tot }}\right)_{\text {test, }}$, Total (endogenous + exogenous) AUC for test article; (AUC Total AUC for reference; (AUC $\left.{ }_{\text {exo }}\right)_{\text {test }}$, AUC for exogenous test article; (AUC , $A \cup C$ for exogenous reference; $A \cup C_{\text {endo, }}$, endogenous $A \cup C ; A \cup C_{\text {tot }}$, total (endogenous + exogenous) AUC

* indicates ratios of $\left(A \cup C_{\text {tot }}\right)_{\text {test }} /\left(A \cup C_{\text {tot }}\right)_{\text {ref }}$ which meet BE criteria when total $A U C$ ratio is used but do not meet $B E$ criteria when exogenous $A U C$ ratio is used.

Table 3: Ratio of AUC for total test article (endogenous + exogenous) to AUC for total reference material (endogenous + exogenous), $\left(A \cup C_{\text {tot }}\right)_{\text {test }} /\left(A \cup C_{\text {tot }}\right)_{\text {ref }}$.

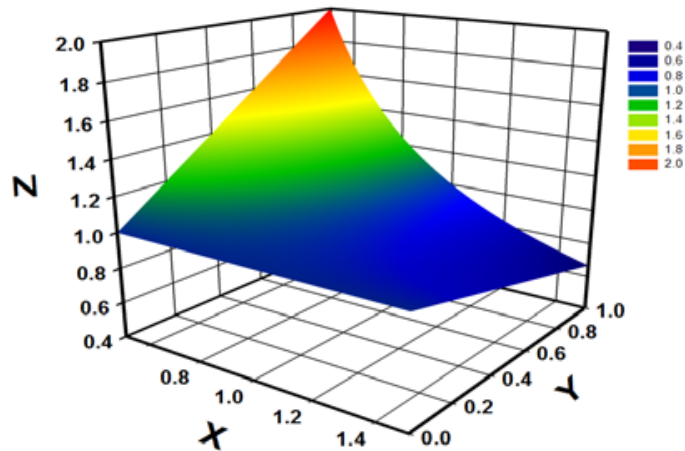

Figure 4: Effect of endogenous AUC contribution to the total AUC in bioequivalence determination

$X$, exogenous $A \cup C$ ratio $=(A \cup C e x o)$ test $/(A \cup C$ exo $)$ ref

$\mathrm{Y}$, Ratios of endogenous $\mathrm{AUC}$ to total $\mathrm{AUC}=\mathrm{AUCendo} /[\mathrm{AUCendo}+(\mathrm{AUCexo})$ ref]

$\mathrm{Z}$, Total AUC ratio/exogenous AUC ratio = [(AUCtot)test $/[\mathrm{AUCtot}) \mathrm{ref}] /[(\mathrm{AUCexo})$ test /(AUCexo)ref]

\begin{tabular}{|c|c|c|c|c|}
\hline Baseline & PK Parameter & Ratio of LSM (\%) & $90 \% \mathrm{Cl}(\%)$ & Bioequivalence \\
\hline \multirow{2}{*}{ Unadjusted } & AUC & 91.3 & $87.2-95.5$ & \multirow{2}{*}{ Yes } \\
\cline { 2 - 4 } & $\mathrm{C}_{\max }$ & 89.3 & $86.1-92.5$ & \\
\hline \multirow{2}{*}{ Adjusted } & AUC & 70.4 & $60.5-82.0$ & \multirow{2}{*}{ No } \\
\cline { 2 - 4 } & $\mathrm{C}_{\max }$ & 81.3 & $75.0-87.9$ & \\
\hline
\end{tabular}

Table 4: Bioequivalence test of tranferrin bound iron following IV administration of iron sucrose.

Inhalation Insulin Determined Using Insulin and Glucose Levels Following Subcutaneous and Inhalation Administration in Humans by Cook et al. [5].

\section{Chiral assay for racemic drugs}

Many marketed and investigational drugs have a chiral center but are administered as racemates. The importance of a chiral assay in pharmacokinetic, pharmacodynamic and drug-drug interaction 

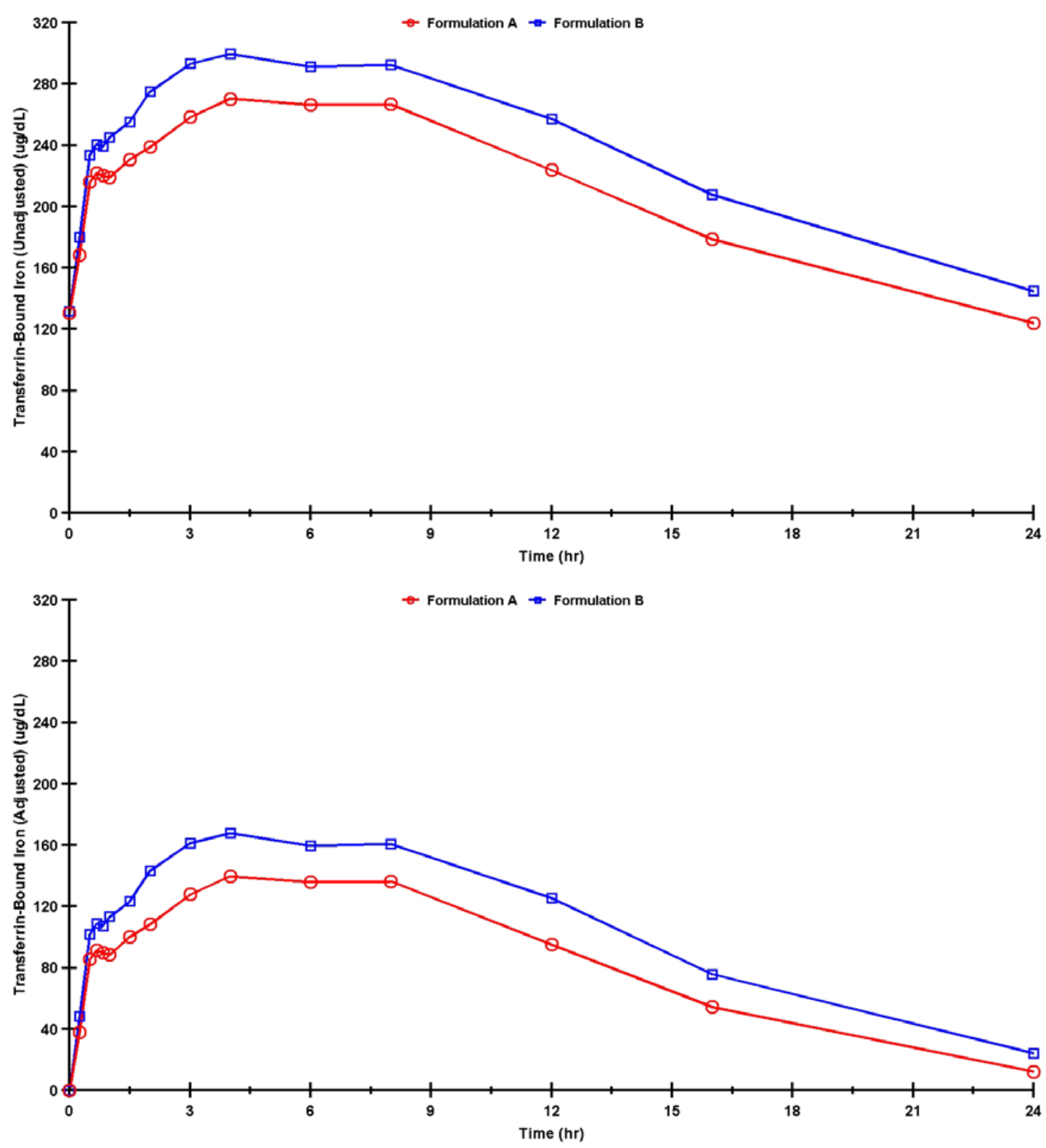

Figure 5: CConcentrations of baseline unadjusted (Upper panel) and adjusted Bottom panel) transferrin bound iron (TBI) concentrations (unpublished data).

studies of racemate drugs has been recognized. However, use of the chiral assay in comparative BA/BE studies has been controversial. A systemic approach to categorize racemate drugs which may need a chiral assay in determining BA/BE following oral administration has been previously proposed [6-10]. In these proposals, emphasis on use of an algorithm for deciding whether a chiral assay should be used or not was based on the enantiospecific first pass metabolism. Two categories of drugs were recommended to use a chiral assay for $\mathrm{BA} / \mathrm{BE}$ determination $[7,10]$. The first category is when a racemic drug undergoes significant first pass stereoselective metabolism. As such, where the first pass metabolism of the active isomer is low and the specific isomer ratio is important, a chiral assay of each isomer is recommended. The second category is when the first pass metabolism of an active isomer is high and there are significant oral-input related changes in the isomer ratio. In this instance, a chiral assay of active isomer as well as non-chiral assay of total drug is recommended.For the non-oral route a chiral assay is generally not required. However, when the active isomer is much more extensively metabolized compared with inactive isomer, and pharmacological activity and/or toxicity is only with the extensively metabolized isomer, a chiral assay of the active isomer as well as the total assay may be required.

\section{Total vs. free concentrations}

Plasma protein binding is readily reversible and relatively constant in therapeutic concentration ranges for most drugs. However, some drugs are extensively plasma protein bound and the binding is concentration dependent in the range of therapeutic concentrations. In this case pharmacokinetics determined using total plasma concentrations will be dose-dependent, whereas pharmacokinetics of free concentrations may be linear $[11,12]$. Concentration-dependent plasma protein binding alone may not require measurement of free concentrations for $\mathrm{BA}$ and $\mathrm{BE}$ determination, as long as similar doses are administered and similar concentration ranges are compared between test and reference formulations. Determination of free concentrations of extensively plasma protein bound drugs may be very difficult because of the low free fraction of drugs and may prove problematic for accurate assessment of $\mathrm{BA} / \mathrm{BE}$. On the other hand, for $\mathrm{BA} / \mathrm{BE}$ assessment of liposome or protein encapsulated formulations and nanosuspensions following an IV dose administration the 


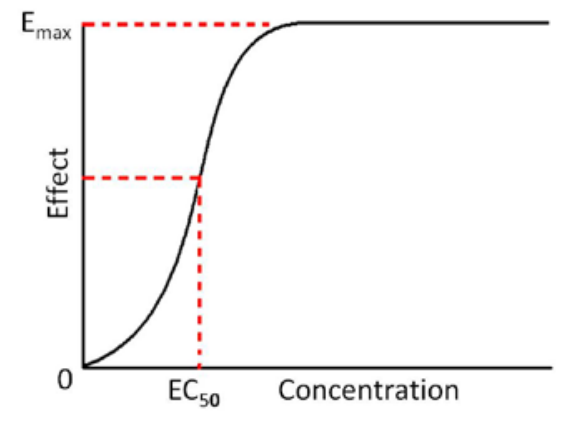

Figure 6: Simulated concentration-effect curve according to Hill's equation.

bioanalytical method should be capable of measuring encapsulated and unencapsulated or total and free drug substance. If a method that distinguishes between encapsulated and unencapsulated drug substance cannot be developed, a justification as to why it is not feasible to develop such a method should be provided. A draft guidance issued by the FDA for $\mathrm{BA}$ and $\mathrm{BE}$ assessment of liposomal formulations is currently available for reference [13,14]. Validated bioanalytical methods should always be used when evaluating the pharmacokinetics and bioavailability of a drug substance.

\section{Acknowledgement}

The author would like to thank Mr. P. Valaitis for his excellent technical assistance, review and valuable comments for this manuscript.

\section{References}

1. FDA Guidance (2002) 21 C.F.R. $§ 320.22$ Criteria for waiver of evidence of in vivo bioavailability or bioequivalence. 57 FR 17998, Apr. 28, 1992, as amended at 67 FR 77673, Dec. 19, 2002.
2. Danielson BG (2004) Structure, chemistry and pharmacokinetics of intravenous iron agent. J Am Soc Nephrol 15:S93-S98.

3. Unpublished data.

4. Statland BE, Winkel $P$ (1977) Relation of day-to-day variation of serum iron concentrations to iron-binding capacity in healthy young women. Am J Clin Pathol 67: 84-90.

5. Cook CS, Valaitis PW, Brugger A, Heise T, Gass J, et al. (2011) Differences in Relative Bioavailability (BA) of Inhalation Insulin Determined using Insulin and Glucose Levels Following Subcutaneous and Inhalation Administration in Humans. J Bioequiv Availab Submitted (2011)

6. Srinivas NR, Barbhaiya RH, Midha KK (2001) Enantiomeric drug development: Issues, considerations, and regulatory requirements. J Pharm Sci 90: 12051215.

7. Midha KK, McKay G, Rawson MJ, Hubbard JW (1998) The impact of stereoisomerism in bioequivalence studies. J Pharm Sci 87: 797-802.

8. Karim A (1996) Enantioselective assays in comparative bioavailability studies of racemic drug formulations: nice to know or need to know? J Clin Pharmacol 36: $490-499$

9. Mehvar R, Jamali F (1997) Bioequivalence of chiral drugs. Clin Pharmacokine 33: 122-141.

10. Karim A, Madhu C, Cook C (2004) Bioequivalency Determination of Racemic Drug Formulations: Is Stereospecific Assay Essential? In Chirality in Drug Design and Development ed. by Reddy IK and Mehvar R.

11. Boudinot FD, Williams RJ, Smith JA (1990) Effect of non-linear plasma protein binding on unbound and total plasma phenylbutazone concentrations in cows. $J$ Vet Pharmacol Ther 13: 132-136.

12. Frey FJ, Gambertoglio JG, Frey BM, Benet LZ, Amend WJ (1982) Nonlinear plasma protein binding and haemodialysis clearance of prednisolone. Eur $J$ Clin Pharmacol 23: 65-74.

13. Draft guidance for industry: Liposome drug products chemistry, manufacturing and controls; human pharmacokinetics and bioavailability;and labeling documentation, FDA. http://www.fda.gov/downloads/Drugs/Guidance Compliance Regulatory Information/Guidances/ucm070570.pdf

14. Internet at http://www.fda.gov/cder/guidance/index.htm 